\title{
The Value of the Model and Quantitative Parameters of Contrast-Enhanced Ultrasound in Judging the Severity of SHPT
}

\author{
Xing-xin Liang, ${ }^{1}$ Fan Li, ${ }^{2}$ Feng Gao, ${ }^{2}$ Yang Liu, ${ }^{2}$ Xiao-hui Qiao, \\ Zheng Zhang, ${ }^{3}$ and Lian-fang $\mathrm{Du}^{2}$ \\ ${ }^{1}$ Department of Medical Ultrasound, Jiangwan Hospital, Hongkou District, Shanghai, China \\ ${ }^{2}$ Department of Medical Ultrasound, Shanghai General Hospital, Shanghai Jiao Tong University School of Medicine, Shanghai, China \\ ${ }^{3}$ Department of Medical Nephrology, Shanghai General Hospital, Shanghai Jiao Tong University School of Medicine, Shanghai, China
}

Correspondence should be addressed to Lian-fang Du; du_lf@163.com

Received 16 September 2016; Revised 6 November 2016; Accepted 23 November 2016

Academic Editor: Ken-ichi Aihara

Copyright (C) 2016 Xing-xin Liang et al. This is an open access article distributed under the Creative Commons Attribution License, which permits unrestricted use, distribution, and reproduction in any medium, provided the original work is properly cited.

\begin{abstract}
Using the model and quantitative parameters of contrast-enhanced ultrasound (CEUS) to assess the severity of secondary hyperparathyroidism (SHPT) was proposed. 42 SHPT patients who underwent CEUS examination were divided into three groups, light, moderate, and heavy as per parathyroid hormone (PTH). The process of CEUS was divided into two phases, wash-in phase and wash-out phase. The three groups were analyzed with their enhancing model in the two phases. The quantitative parameters of CEUS such as Arrival Time (AT), Time to Peak (TTP), Mean Transit Time (MTT), and Maximum Intensity (IMAX) were measured by time-intensity curve (TIC) and compared among the three groups. The enhancing model of light SHPT, moderate SHPT, and heavy SHPT showed statistical significance in wash-in phase and wash-out phase $(P<0.05)$. No difference was observed in AT and TTP among the three groups $(P>0.05)$ while MTT and IMAX showed statistical significance $(P<0.05)$. The CEUS of light SHPT was characterized by "slow-in, fast-out, and lower-enhancement" with short enhancement time; the CEUS of moderate SHPT was characterized by "fast-in, fast-out, and higher-enhancement" with slightly long enhancement time; the CEUS of heavy SHPT was characterized by "fast-in, slow-out, and higher-enhancement" with long enhancement time. Therefore, the model and quantitative parameters of CEUS can be benefit for the assessment of the severity of SHPT.
\end{abstract}

\section{Introduction}

Secondary hyperparathyroidism (SHPT) is ubiquitous in patients with chronic renal failure (CRF) treated by long-term dialysis [1], Hypocalcemia, phosphate retention, and 1,25dihydroxycholecalciferol $\left(1,25(\mathrm{OH})_{2} \mathrm{D}_{3}\right)$ deficiency lead to the synthesis and release of parathyroid hormone (PTH) and the persistent over stimulation of parathyroid gland (PTG). In particular in severe SHPT, PTG becomes less responsive to hormonal stimuli which trigger cell hypertrophy-hyperplasia and leads to tertiary or autonomous hyperparathyroidism. Severe SHPT can cause severe clinical symptoms such as osteoporosis, pathological fracture, and other skeletal system diseases [2]; recurrent urinary stones and other urinary system diseases [3]; depression, anxiety, and other nonspecific mental symptoms [4]; insomnia, fatigue, high blood pressure, and other cardiovascular system diseases [5]; anorexia, nausea, long-term constipation, and other digestive system diseases [6]; serious anemia $[7,8]$.

Severity of SHPT has been evaluated mainly by the level of PTH, but hypertrophy (increased cell volume) and hyperplasia (increased cell number) are the pathological basis of the increase of PTH. So, carrying out imaging examination will possibly find parathyroid hyperplasia earlier than the increase of PTH and will possibly indicate the progress of parathyroid hyperplasia earlier than the increase of PTH, which will lead to the diagnosis and treatment of SHPT from passive waiting to active predicting. Conventional ultrasound plays a pivotal role in the diagnosis and follow-up of SHPT. However, its sensitivity of assessing the severity of SHPT is not high; it has a reported sensitivity of $45-70 \%$ [9]. Therefore, CEUS is evaluated for assessing the severity of SHPT, based on its dynamic microcirculation. 


\section{Methods and Materials}

2.1. Subjects. This study was conducted within the Department of Ultrasound at Shanghai General Hospital between May 2014 and December 2014. Ultrasonography of PTGs was performed in 50 patients whose PTH level was $\geq 250 \mathrm{mg} / \mathrm{dL}$ (PTH level $\geq 250 \mathrm{mg} / \mathrm{dL}$ defined as SHPT). Of the 50 patients, 8 patients were not found to have parathyroid hyperplasia on ultrasonography. We excluded these 8 patients. Thus, 42 patients had been incorporated into the present study. Serum iPTH levels were determined by immunoradiometric assay.

The 42 patients were divided into three groups: light SHPT (iPTH rang $\geq 250 \mathrm{ng} / \mathrm{L}<600 \mathrm{ng} / \mathrm{L}$ ), moderate SHPT (iPTH range $\geq 600 \mathrm{ng} / \mathrm{L}<800 \mathrm{ng} / \mathrm{L}$ ), and heavy SHPT (iPTH range $\geq 800 \mathrm{ng} / \mathrm{L}) .12$ patients were light SHPT patients (7 men, 5 women) with mean age of $56.12 \pm 8.56$ years (range, 29 81 years); 18 patients were moderate SHPT (9 men, 9 women) with mean age of $57.72 \pm 6.24$ years (range, $26 \sim 79$ years); 12 patients were heavy SHPT (5 men, 7 women) with mean age of $54.19 \pm 6.86$ years (range, $31 \sim 80$ years). 12 patients with heavy SHPT had undergone parathyroidectomy and achieved pathologic results.

2.2. Apparatus. Sequoia 512 (Siemens, Germany) ultrasound scanner with a $4 \sim 9 \mathrm{MHz}$ linear probe was adopted to conduct CEUS examination. Mechanical Index (MI) was set to 0.20 and contrast agent was six sulphur hexafluoride microbubbles (SonoVue, Bracco Corporation, Italy). Before application, we injected $5 \mathrm{~mL}$ normal saline into SonoVue powder, oscillated it forcibly, and shocked it fully and then selected $3 \mathrm{~mL}$ to bolus injection through elbow vein. In the imaging process, it was necessary to ask patients to avoid swallowing and keep quiet breathing. The observation time was no less than 3 min. Injection was done by the same assistant in all cases. All CEUS examinations were performed by one experienced examiner who engaged in parathyroid examination for more than 5 years. Image analysis was performed by two expert sinologists who were blinded to the knowledge of patients' symptoms and iPTH.

2.3. Study Design. CEUS examination had been authorized by the Ethics Committee of Shanghai General Hospital, and patients signed informed consent before inspection.

The CEUS investigation was divided into two phases: wash-in phase and wash-out phase. The wash-in phase ranged from $0 \mathrm{~s}$ to $30 \mathrm{~s}$ after application of the contrast agent. The wash-out phase ranged from $30 \mathrm{~s}$ to $120 \mathrm{~s}$ after application of the contrast agent.

The whole imaging process was stored and analyzed by SonoLiver (TomTec, Germany) software. Arrival Time (AT), Time to Peak (TTP), Mean Transit Time (MTT), and Maximum Intensity (IMAX) were measured by time-intensity curve (TIC). AT was defined as the time interval from application time to the time when the gland's intensity reached $10 \%$. TTP was defined as the time interval from application time to the time when the gland's intensity reached peak. MTT was defined as the time interval from Arrival Time to the time when the peak intensity fell to half. IMAX was defined as the gland's peak intensity. We performed CEUS examination on the largest gland of PTGs and we took their average when the size of glands was similar.

Because the size of parathyroid glands is known to be correlated with the severity of SHPT and there is little doubt that in glands larger than $0.5 \mathrm{~cm}^{3}$, nodular hyperplasia probably exists [10], we also divided the 42 patients into two groups (volume $<0.5 \mathrm{~cm}^{3}$ and volume $\geq 0.5 \mathrm{~cm}^{3}$ ) and examined the relationship between the size of glands and the enhancing model of CEUS. PTG volume was calculated according to the formula $V=(a \times b \times c) \times \Pi / 6$.

2.4. Statistical Analysis. IBM SPSS Analytics 16.0 software (Chicago III, USA) was used for statistical analysis. The measurement data was displayed as mean \pm standard error and analyzed by applying the Analysis of Variance (ANOVA), and $P$ values of $<0.05$ were regarded statistically significant. Enumeration data was analyzed by applying the $\chi^{2}$ test and the Cochran-Armitage Trend test, and $P$ values of $<0.05$ were regarded statistically significant.

\section{Results}

3.1. CEUS Manifestation. The differences of the position of parathyroid glands among the three groups were statistically trivial $(P=0.11)$, as shown in Table 1 .

The differences of the enhancing model of CEUS among the three groups were statistically significant $(P<0.05)$, as shown in Table 2.

The differences of the enhancing model of CEUS between the different volumes were statistically significant $(P<0.05)$, as shown in Table 3.

3.2. Quantitative Parameters of CEUS. As shown in Table 4, the quantitative parameters of CEUS were compared among the three groups: no difference was observed among the three groups in AT and TTP $(P>0.05)$ while MTT and IMAX showed statistical significance among the three groups $(P<$ 0.05).

3.3. Surgical Histopathology. The histopathology of 12 cases (surgical patients) was as follows: 12 patients all had nodular hyperplasia.

\section{Discussion}

SHPT is a disorder related to mortality $[11,12]$; it is not only a state of increased PTH synthesis and secretion, but more importantly a state of parathyroid hyperplasia. Numerous studies have shown that SHPT patients exhibit signs of hyperplasia very early, first diffuse, and later nodular hyperplasia [10]. Homogeneous echoes and smaller enlargement were often found in diffuse hyperplasia with less vascularity which can apply the calcitriol [13] to inhibit the secretion of PTH. Inhomogeneous echoes and larger enlargement were often found in nodular hyperplasia with paranodular vascularity [14] which must undergo parathyroidectomy because of its resistance to drug.

Conventional ultrasound is simple, accurate, noninvasive, and reproducible. It can clearly show the location of 
TABLE 1: The position of parathyroid glands of three groups.

\begin{tabular}{lcccc}
\hline Position & \multicolumn{1}{c}{ Groups } & & \\
& Light SHPT $(N=12)$ & Moderate SHPT $(N=18)$ & Heavy SHPT $(N=12)$ & 7 \\
Superior & 4 & 10 & 5 & 0.11 \\
Inferior & 8 & 8 & 5 \\
\hline
\end{tabular}

Note. All data were analyzed by applying the Cochran-Armitage Trend test.

TABLE 2: The enhancing model of CEUS of three groups in two phases.

\begin{tabular}{|c|c|c|c|c|c|}
\hline \multirow{2}{*}{\multicolumn{2}{|c|}{ Phases }} & \multicolumn{3}{|c|}{ Groups } & \multirow{2}{*}{$P$ value } \\
\hline & & Light SHPT $(N=12)$ & Moderate SHPT $(N=18)$ & Heavy SHPT $(N=12)$ & \\
\hline \multirow{2}{*}{ Wash-in phase } & High enhanced & 1 & 14 & 11 & \multirow{2}{*}{$<0.05$} \\
\hline & Low enhanced & 11 & 4 & 1 & \\
\hline \multirow{2}{*}{ Wash-out phase } & High enhanced & 2 & 5 & 11 & \multirow{2}{*}{$<0.05$} \\
\hline & Low enhanced & 10 & 13 & 1 & \\
\hline
\end{tabular}

Note. All data were analyzed by applying the Cochran-Armitage Trend test.

TABLE 3: The enhancing model of CEUS of different volumes in two phases.

\begin{tabular}{lcccc}
\hline & & & Groups & \\
& Phases & Volume $<0.5 \mathrm{~cm}^{3}(N=27)$ & Volume $\geq 0.5 \mathrm{~cm}^{3}(N=15)$ & 15 \\
Wash-in phase & High enhanced & 11 & 0 & $<0.05$ \\
& Low enhanced & 16 & 15 & $<0.05$ \\
\multirow{2}{*}{ Wash-out phase } & High enhanced & 3 & 24 & 0 \\
\hline
\end{tabular}

Note. All data were analyzed by applying the $\chi^{2}$ test.

TABLE 4: Quantitative parameters of CEUS between the three groups.

\begin{tabular}{|c|c|c|c|c|}
\hline \multirow{2}{*}{ Parameter } & \multicolumn{3}{|c|}{ Group } & \multirow{2}{*}{$P$ value } \\
\hline & Light SHPT $(N=12)$ & Moderate SHPT $(N=18)$ & Heavy SHPT $(N=12)$ & \\
\hline $\mathrm{AT}(\mathrm{s})$ & $5.04 \pm 0.51$ & $4.91 \pm 0.34$ & $4.76 \pm 0.30$ & 0.23 \\
\hline TTP(s) & $10.91 \pm 0.71$ & $11.38 \pm 0.78$ & $11.62 \pm 0.67$ & 0.062 \\
\hline MTT(s) & $9.45 \pm 1.48$ & $12.91 \pm 0.85$ & $14.58 \pm 1.21$ & $<0.05$ \\
\hline $\operatorname{IMAX}(\mathrm{dB})$ & $0.28 \pm 0.08$ & $1.82 \pm 0.22$ & $2.61 \pm 0.40$ & $<0.05$ \\
\hline
\end{tabular}

Note. All data were analyzed by applying the ANOVA.

hyperplastic glands and their adjacent tissues and organs. So it is the first choice for diagnosing SHPT. But conventional ultrasound has low sensitivity in assessing the severity of SHPT because it cannot display the dynamic perfusion characteristics of PTGs. CEUS can make up for the deficiency of conventional ultrasound and can detect nodular hyperplasic parathyroid glands before the occurrence of hyperparathyroidism which is resistant to medical therapy because a major feature of CEUS is the highly dynamic visualization of vascularization and blood flow of the tissue. CEUS with low acoustic power can better detect the microcirculation of PTGs and can dynamically detect the capillary perfusion of PTGs, which is more close to the pathologies. SonoVue is already known to allow continuous imaging at low acoustic power, providing an easier and more accurate depiction of microcirculation, which is not assessable by means of conventional ultrasound [15-17].
Based on our study, of 12 cases of light SHPT, 10 cases showed the enhancing model of "slow-in and fast-out"; of 18 cases of moderate SHPT, 13 cases showed the enhancing model of "fast-in and fast-out"; of 12 cases of heavy SHPT, 11 cases showed the enhancing model of "fast-in and slow-out." The enhancing model of light SHPT and moderate SHPT and heavy SHPT showed statistical significance in washin phase and wash-out phase $(P<0.05)$. However, of 12 cases of light SHPT, 1 case showed the enhancing model of "fast-in and slow-out"; of 18 cases of moderate SHPT, 1 case showed the enhancing model of "fast-in and slow-out"; of 12 cases of heavy SHPT, 1 case showed the enhancing model of "slow-in and fast-out". So, there were a few overlaps in CEUS manifestations among the three groups, which depended on quantitative analysis of CEUS.

Based on our study, no difference was observed in AT and TTP among the three groups $(P>0.05)$ while MTT 

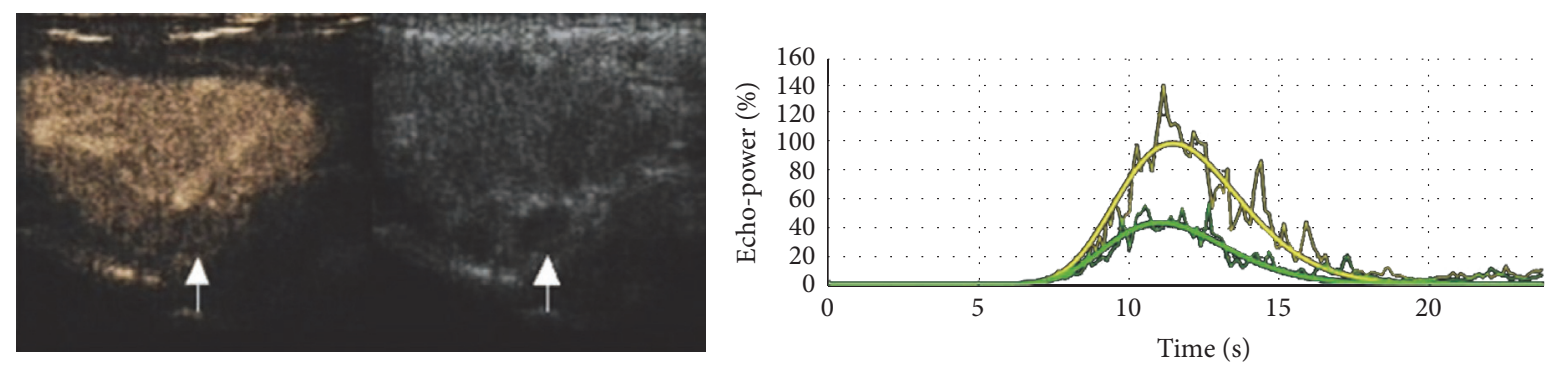

FIgURE 1: The CEUS of light SHPT. The CEUS of light SHPT is characterized by "slow-in, fast-out, and lower-enhancement" with short enhancement time.
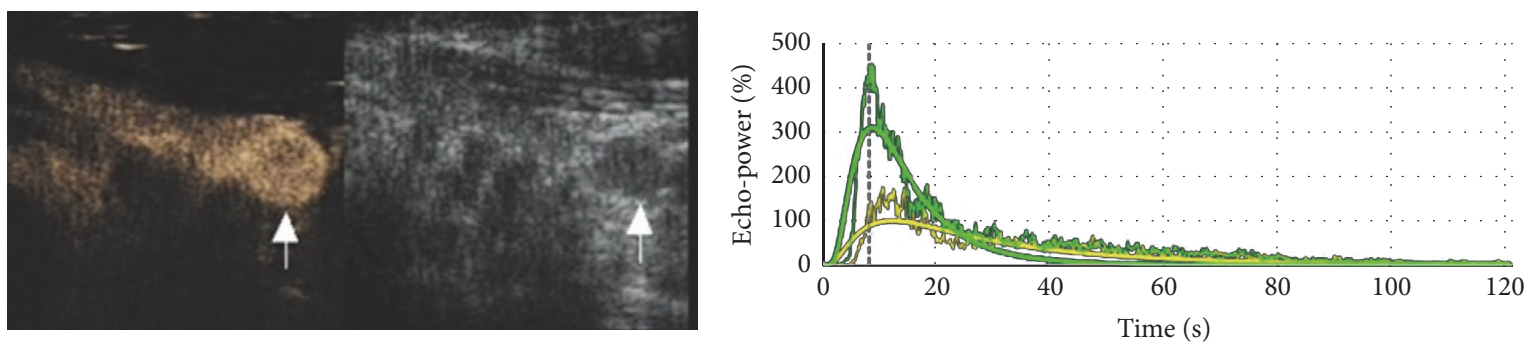

FIGURE 2: The CEUS of moderate SHPT. The CEUS of moderate SHPT is characterized by "fast-in, fast-out, and higher-enhancement" with slightly long enhancement time.
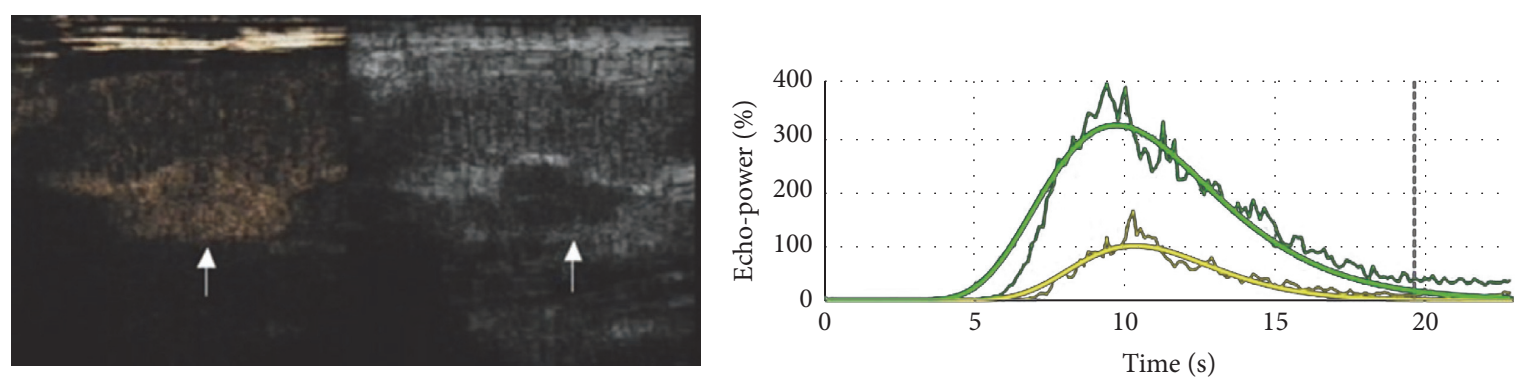

FIGURE 3: The CEUS of heavy SHPT. The CEUS of heavy SHPT is characterized by "fast-in, slow-out, and higher-enhancement" with long enhancement time.

and IMAX showed statistical significance $(P<0.05)$. With the aggravation of the severity of SHPT, MTT was getting longer and longer; IMAX was getting higher and higher. These differences may be related to the characteristics of capillary perfusion and determined by different pathological characteristics of the three groups; the perfusion and duration of microcirculation increase with the aggravation of the severity of SHPT.

In our study, the differences of the position of PTGs among the three groups were statistically trivial $(P=0.11)$, so the position of PTGs does not affect the results of the enhancing model of CEUS. Although the differences of the enhancing model of CEUS between the different volumes were statistically significant, but when volume was $<0.5 \mathrm{~cm}^{3}$, there were too many CEUS manifestations which could not classify the enhancing model of different severity of SHPT, so this study took the classification of iPTH.

Within the PTG, calcifications or cysts can be detected. In patients with such glands, PTH is significantly elevated [18]. Four of the 12 patients in heavy SHPT have cystic changes including the one with volume of smaller than $0.5 \mathrm{~cm}^{3}$ and less vascularity, so its PTH was elevated but its enhancing model was "low enhanced" in wash-in phase and wash-out phase.

In conclusion, our results showed that the CEUS of light SHPT was characterized by "slow-in, fast-out, and lowerenhancement" with short enhancement time (Figure 1); the CEUS of moderate SHPT was characterized by "fast-in, fastout, and higher-enhancement" with slightly long enhancement time (Figure 2); the CEUS of heavy SHPT was characterized by "fast-in, slow-out, and higher-enhancement" with long enhancement time (Figure 3). The model and quantitative parameters of CEUS can be benefit for the assessment of the severity of SHPT.

\section{Competing Interests}

The authors declare that there is no conflict of interests regarding the publication of this paper. 


\section{References}

[1] Q. He, D. Zhuang, L. Zheng et al., “Total parathyroidectomy with trace amounts of parathyroid tissue autotransplantation as the treatment of choice for secondary hyperparathyroidism: a single-center experience," BMC Surgery, vol. 14, article no. 26, 2014.

[2] C. Marcocci, L. Cianferotti, and F. Cetani, "Bone disease in primary hyperparathyrodism," Therapeutic Advances in Musculoskeletal Disease, vol. 4, no. 5, pp. 357-368, 2012.

[3] F. V. D. de la Guardia, M. A. Martin, M. A. A. Polo, S. Q. Flores, J. L. M. Ortiz, and A. Z. Gomez, "Renal lithiasis in patients with primary hyperparathyroidism. Evolution and treatment," Archivos Españoles de Urología, vol. 63, no. 1, pp. 32-40, 2010.

[4] T. Weber, J. Eberle, U. Messelhäuser et al., "Parathyroidectomy, elevated depression scores, and suicidal ideation in patients with primary hyperparathyroidism: results of a prospective multicenter study," Archives of Surgery, vol. 148, no. 2, pp. 109115, 2013.

[5] D. Han, S. Trooskin, and X. Wang, "Prevalence of cardiovascular risk factors in male and female patients with primary hyperparathyroidism," Journal of Endocrinological Investigation, vol. 35, no. 6, pp. 548-552, 2012.

[6] A. Ragno, J. Pepe, D. Badiali et al., "Chronic constipation in hypercalcemic patients with primary hyperparathyroidism," European Review for Medical and Pharmacological Sciences, vol. 16, no. 7, pp. 884-889, 2012.

[7] R. Schneider, G. Kolios, B. M. Koch, E. D. Fernández, D. K. Bartsch, and K. Schlosser, "An economic comparison of surgical and medical therapy in patients with secondary hyperparathyroidism - the German perspective," Surgery, vol. 148, no. 6, pp. 1091-1099, 2010.

[8] S. Iannazzo, M. Carsi, and S. Chiroli, "A cost-utility analysis of cinacalcet in secondary hyperparathyroidism in five european countries," Applied Health Economics and Health Policy, vol. 10, no. 2, pp. 127-138, 2012.

[9] T. Defechereux and M. Meurisse, "Renal hyperparathyroidism: current therapeutic approaches and future directions," Operative Techniques in Otolaryngology-Head and Neck Surgery, vol. 20, no. 1, pp. 71-78, 2009.

[10] M. Fukagawa, "Cell biology of parathyroid hyperplasia in uremia," American Journal of the Medical Sciences, vol. 317, no. 6, pp. 377-382, 1999.

[11] G. A. Block, P. S. Klassen, J. M. Lazarus, N. Ofsthun, E. G. Lowrie, and G. M. Chertow, "Mineral metabolism, mortality, and morbidity in maintenance hemodialysis," Journal of the American Society of Nephrology, vol. 15, no. 8, pp. 2208-2218, 2004.

[12] M. L. Melamed, J. A. Eustace, L. Plantinga et al., "Changes in serum calcium, phosphate, and PTH and the risk of death in incident dialysis patients: a longitudinal study," Kidney International, vol. 70, no. 2, pp. 351-357, 2006.

[13] I. G. Nikolov, A. Mozar, T. B. Drüeke, and Z. A. Massy, "Impact of disturbances of calcium and phosphate metabolism on vascular calcification and clinical outcomes in patients with chronic kidney disease," Blood Purification, vol. 27, no. 4, pp. 350-359, 2009.

[14] D. Pavlovic and H. T. Brzac, "Ultrasonographic evaluation of parathyroid hyperplasia in dialysis patients," TheScientificWorldJournal, vol. 6, pp. 1599-1608, 2006.
[15] C. Greis, "Ultrasound contrast agents as markers of vascularity and microcirculation," Clinical Hemorheology and Microcirculation, vol. 43, no. 1-2, pp. 1-9, 2009.

[16] K. Stock, C. H. von Weyhern, J. Slotta-Huspenina et al., "Microcirculation of subepithelial gastric tumors using contrastenhanced ultrasound," Clinical Hemorheology and Microcirculation, vol. 45, no. 2-4, pp. 225-232, 2010.

[17] P. Zengel, V. Siedek, A. Berghaus, and D. A. Clevert, "Intraductally applied contrast-enhanced ultrasound (IA-CEUS) for improved visualization of obstructive diseases of the salivary glands, primary results," Clinical Hemorheology and Microcirculation, vol. 45, no. 2-4, pp. 193-205, 2010.

[18] H. Tomic-Brzac and D. Pavlovic, "Possibility of 2-D and Color Doppler sonography in distinguishing diffuse and nodular parathyroid hyperplasia," Ultrasound in Medicine and Biology, vol. 26, supplement 2, p. A183, 2000. 


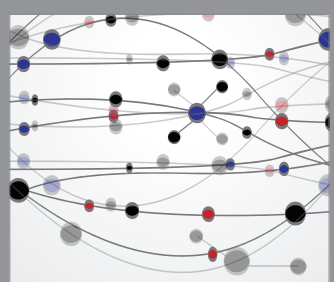

The Scientific World Journal
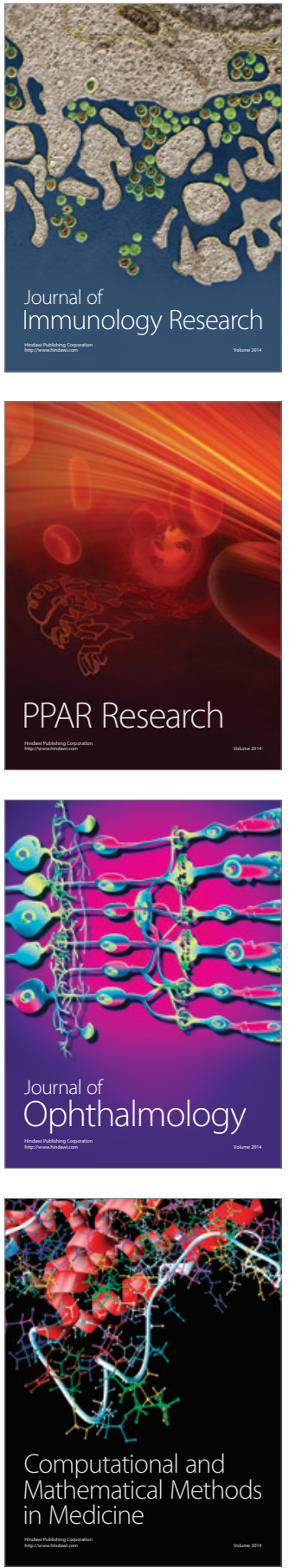

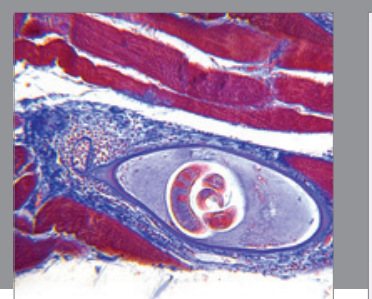

Gastroenterology Research and Practice

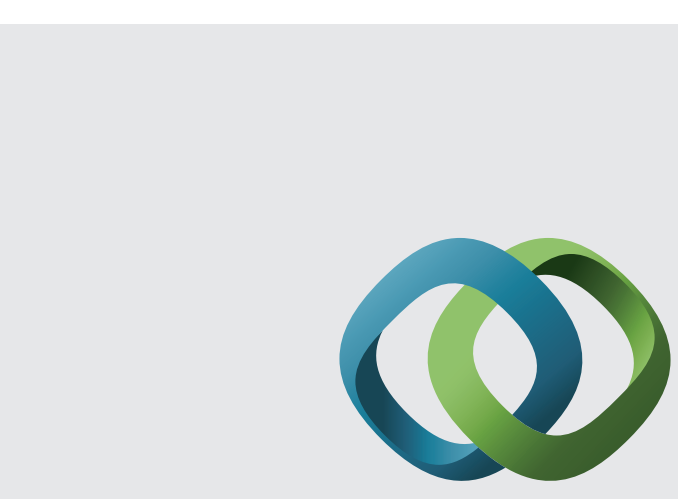

\section{Hindawi}

Submit your manuscripts at

http://www.hindawi.com
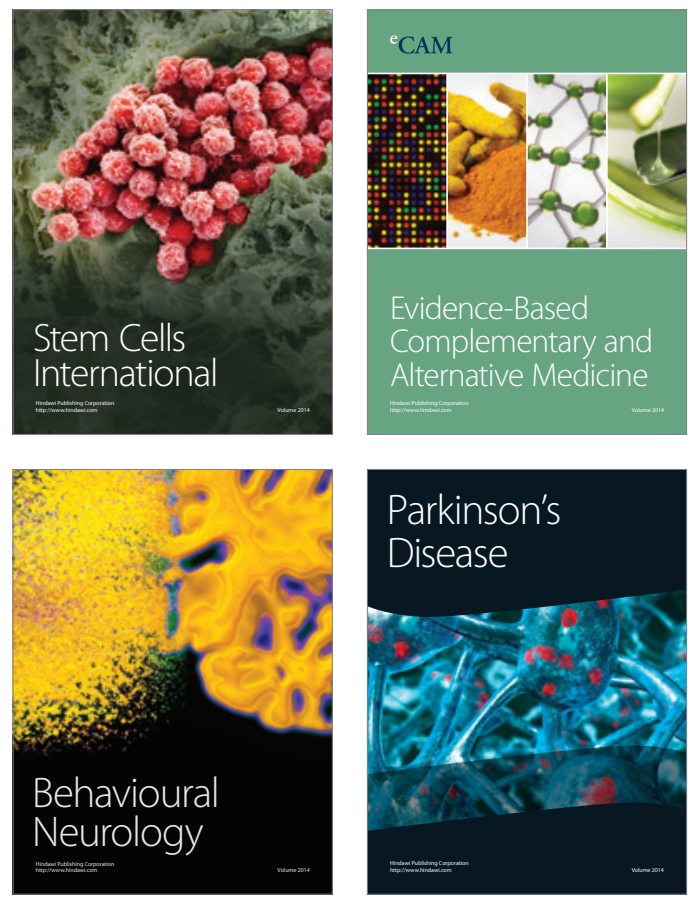
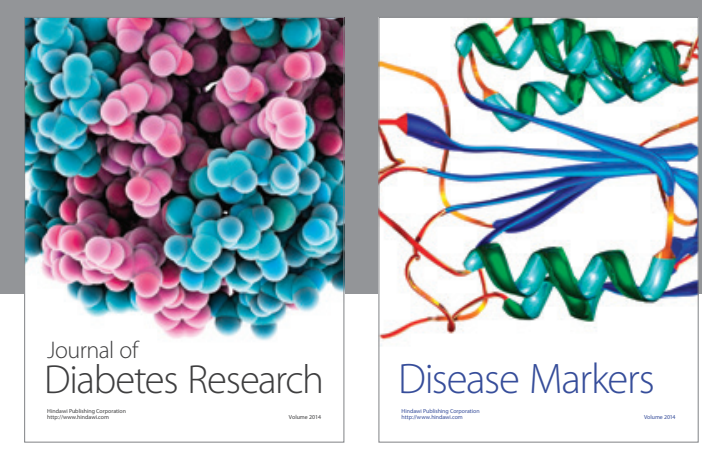

Disease Markers
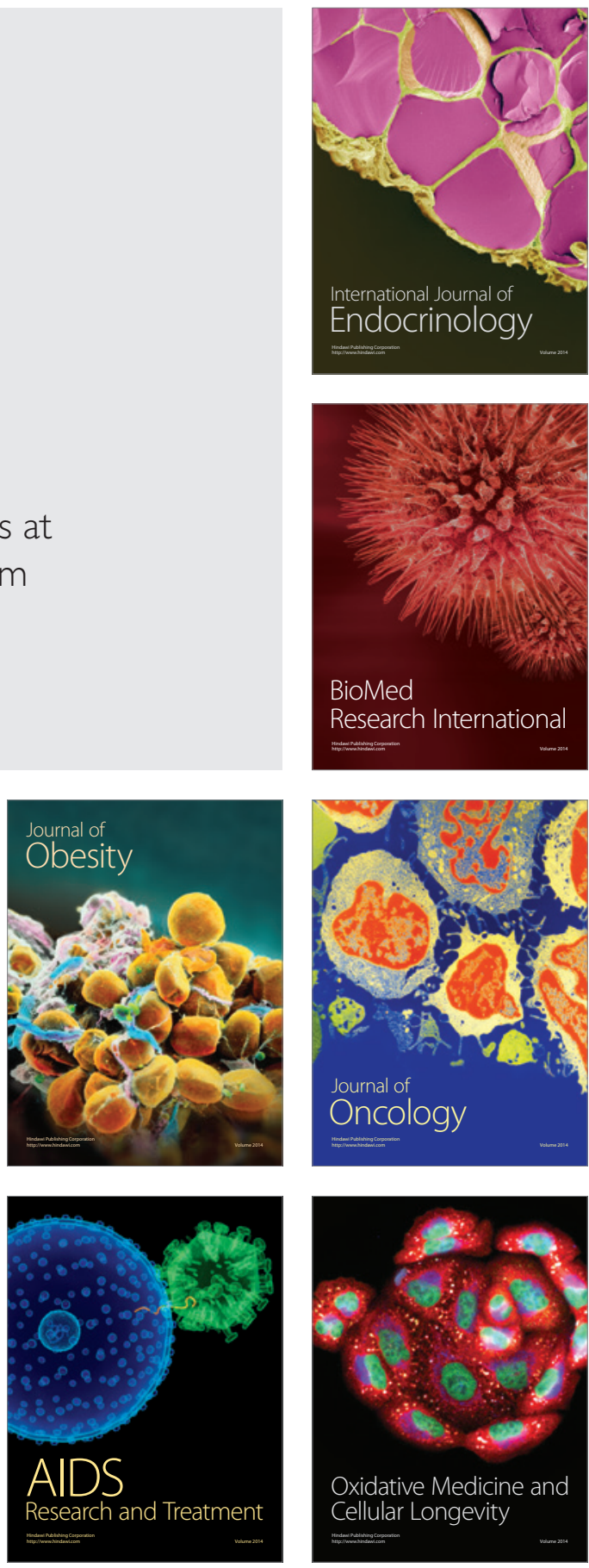